\title{
Optical Imaging of Tumor Response to Hyperbaric Oxygen Treatment and Irradiation in an Orthotopic Mouse Model of Head and Neck Squamous Cell Carcinoma
}

\author{
Joanna A. M. Braks, ${ }^{1}$ Linda Spiegelberg, ${ }^{1}$ Senada Koljenovic, ${ }^{2}$ Yanto Ridwan, ${ }^{3}$ \\ Stijn Keereweer, ${ }^{4}$ Roland Kanaar, ${ }^{3,5}$ Eppo B. Wolvius, ${ }^{1}$ Jeroen Essers ${ }^{3,5,6}$ \\ ${ }^{1}$ Department of Oral and Maxillofacial Surgery, Erasmus Medical Center, PO Box 2040, 3000 CA, Rotterdam, The Netherlands \\ ${ }^{2}$ Department of Pathology, Erasmus Medical Center, PO Box 2040, 3000 CA, Rotterdam, The Netherlands \\ ${ }^{3}$ Department of Genetics, Erasmus Medical Center, PO Box 2040, 3000 CA, Rotterdam, The Netherlands \\ ${ }^{4}$ Department of Otorhinolaryngology and Head \& Neck Surgery, Erasmus Medical Center, PO Box 1738, 3015 CE, Rotterdam, Netherlands \\ ${ }_{5}^{5}$ Department of Radiation Oncology, Erasmus Medical Center, PO Box 2040, 3000 CA, Rotterdam, The Netherlands \\ ${ }^{6}$ Department of Vascular Surgery, Erasmus Medical Center, PO Box 2040, 3000 CA, Rotterdam, The Netherlands
}

\begin{abstract}
Purpose: Hyperbaric oxygen therapy (HBOT) is used in the treatment of radiation-induced tissue injury but its effect on (residual) tumor tissue is indistinct and therefore investigated in this study. Procedures: Orthotopic FaDu tumors were established in mice, and the response of the (irradiated) tumors to HBOT was monitored by bioluminescence imaging. Near infrared fluorescence imaging using AngioSense750 and Hypoxisense680 was applied to detect tumor vascular permeability and hypoxia.

Results: HBOT treatment resulted in accelerated growth of non-irradiated tumors, but mouse survival was improved. Tumor vascular leakiness and hypoxia were enhanced after HBOT, whereas histological characteristics, epithelial-to-mesenchymal transition markers, and metastatic incidence were not influenced.

Conclusions: Squamous cell carcinoma responds to HBOT with respect to tumor growth, vascular permeability, and hypoxia, which may have implications for its use in cancer patients. The ability to longitudinally analyze tumor characteristics highlights the versatility and potential of optical imaging methods in oncological research.
\end{abstract}

Key words: Hyperbaric oxygen therapy, Radiation, Optical imaging, Near infrared fluorescence, Squamous cell carcinoma, Lymph node metastasis, Animal model, Head and neck cancer

Electronic supplementary material The online version of this article (doi:10.1007/s11307-015-0834-8) contains supplementary material, which is available to authorized users.

Correspondence to: Joanna Braks; e-mail: j.braks@erasmusmc.nl, Jeroen Essers; e-mail: j.essers@erasmusmc.nl

\section{Introduction}

$\mathrm{H}$ ead and neck squamous cell carcinoma (HNSCC) is the sixth most common cancer type worldwide and is associated with a poor prognosis. Treatment of these cancers often involves surgical resection followed by radiotherapy. 
Despite advances in radiation protocols that minimize the targeted tissue volume, radiation treatment often causes considerable damage to the surrounding healthy tissues resulting in complications like impaired wound healing and osteoradionecrosis. Hyperbaric oxygen therapy (HBOT) is frequently used in the management of radiation-induced tissue injury, and has shown beneficial effects although its working mechanism has not completely been unraveled yet $[1,2]$. In HBOT, patients inspire $100 \%$ oxygen at elevated barometric pressure, which enhances the amount of oxygen that is dissolved in the plasma leading to an increase of the oxygen tension in tissues. By creating an oxygen gradient, HBOT is thought to induce neovascularization by which the progressive loss of the microvasculature in hypoxic irradiated tissue may be overcome and tissue healing is improved [3-5].

The use of HBOT in patients with a history of cancer has often raised concerns about the promoting effect this therapy might have on the growth of (residual) tumor tissue. Poor oxygenation and abnormal vasculature is a common feature of solid tumors and reduces the ability of cells to divide. It was anticipated that by its pro-angiogenic effect, HBOT would stimulate cancer growth and recurrence [6-8]. On the other hand, tumor hypoxia is known to be essential for the progression of cancer and is related to increased cell survival, induction of angiogenesis, metastasis, and therapy resistance [9, 10]. Enhanced oxygenation of tumors by HBOT could therefore lead to less aggressive cancer growth and a better prognosis. Based on clinical and experimental studies, it was recently adopted that there is no evidence that HBOT has a cancer-promoting effect $[11,12]$. Even more, on certain cancer subtypes like gliomas and mammary tumors, an anti-angiogenic and growth-inhibitory effect of HBOT was reported [13-15].

In most studies in which subcutaneously implanted squamous carcinoma cell lines in mice were used, no differences in growth between control and HBOT groups were seen [16-20]. However, in a recent study, Paniello et al. [21] reported enhanced growth of HNSCC tumor cells in $\mathrm{C} 3 \mathrm{H}$ mice after HBOT. These divergent outcomes suggest that the choice of the experimental model, regarding cancer cell type, tumor location, or HBOT protocol, is critical for the proper determination of tumor responses to HBOT.

One of the tumor conditions that is relevant to the clinical situation but has been scarcely investigated in experimental HBOT studies is the irradiated tumor. Since radiation not only modifies the cancer cells but also the microenvironment of the tumor by affecting angiogenesis and the hypoxic state of the tissue [22], previous irradiation might well influence the response of the residual tumor to HBOT.

By using an improved, clinically relevant tumor model and advanced optical imaging strategies, we intend to increase the insight on the effects of hyperbaric oxygen on tumor growth and factors that influence tumor behavior like tumor vascularization, hypoxia, and differentiation. In the present study, we used bioluminescent imaging (BLI) to non-invasively and adequately monitor the growth of a human squamous cell carcinoma line $(\mathrm{FaDu})$ in the floor of the mouth of immunodeficient mice. Near infrared fluorescence (NIRF) optical imaging was applied to detect and quantify the effects of HBOT and irradiation on specific tumor characteristics in vivo. The fluorescent blood pool agent AngioSense was used to analyze tumor blood vessel quality, and the NIRF targeting probe HypoxiSense was applied to study hypoxia in the tumors. Furthermore, this orthotopic mouse model allowed us to investigate the effects of HBOT on the development of regional and distant metastases, which are likewise frequently seen in patients with HNSCC.

\section{Materials and Methods}

\section{Mice}

All animal experiments of this study were approved by the Animal Experiments Committee of the Erasmus Medical Center (DEC 2645). The Dutch Experiments on Animal Act is established under the European guidelines (EU Directive No. 86/609/EEC regarding the Protection of Animals used for Experimental and Other Scientific Purposes). BALB/c nu/nu female mice (Charles River Laboratories), aged 8 to 11 weeks, were kept in filter-top cages with autoclaved pellet food and sterilized water without restriction. Mice with tumors in the floor of the mouth were given soft food and were monitored daily. Animals were euthanized when they had lost more than $20 \%$ of their initial body weight or had reached day 35 after tumor implantation.

\section{Tumor Generation}

The human hypopharyngeal squamous cell carcinoma line FaDuluc2 was kindly received from the laboratory of Prof. C.W. Löwik, $\mathrm{PhD}$ (Leiden University Medical Center, Leiden, The Netherlands). This cell line had been transfected with a luciferase-expressing vector (pCAGGS- Luc-2) allowing the monitoring of the tumor growth by bioluminescence imaging (BLI) [23]. FaDu-luc2 cells were grown in Dulbecco's modified Eagle's medium (DMEM, Lonza) supplemented with $10 \%(v / v)$ fetal bovine serum (Hyclone) and antibiotics (50 units $/ \mathrm{ml}$ of penicillin and $50 \mu \mathrm{g} / \mathrm{ml}$ streptomycin) at $37^{\circ} \mathrm{C}$ in a humidified atmosphere of $5 \% \mathrm{CO}_{2}$ in air.

Orthotopic tumors were established by transcervical injection of $1 \times 10^{5}$ cultured FaDu-luc2 cells, suspended in $20 \mu \mathrm{l}$ serum-free DMEM into the floor of the mouth of anesthetized (2-4\% isoflurane) nude mice.

\section{Hyperbaric Oxygen Treatment (HBOT)}

Treatment with hyperbaric oxygen started at day 5 after tumor implantation and consisted of daily sessions, until the end of the experiment with a maximum of 30 sessions. The hyperbaric oxygen chamber used in this study was custom-built for small laboratory animals (Hytech BV, Raamsdonksveer, The Netherlands) [24]. Each session started with a compression phase of $15 \mathrm{~min}$, during which the pressure in the chamber was elevated to 2.4 atm absolute (ATA) and the oxygen level to $100 \%$. After $90 \mathrm{~min}$ of isopression, decompression to 1 ATA took place in $15 \mathrm{~min}$. 


\section{Radiation Therapy (RT)}

Mice were anesthetized $(65 \mathrm{mg} / \mathrm{kg}$ ketamine and $10 \mathrm{mg} / \mathrm{ml}$ xylazine) and locally irradiated at day 5 after tumor implantation with a single dose of 5 Gy using a Gammacell 40 Exactor Cs-137 $\gamma$-source. With this dose, tumor growth is significantly reduced, but not all tumor cells are killed. Mice were shielded using a Gammacell 40 Collimator centering the head and neck region in a 3 -cm radiation field.

\section{Bioluminescence Imaging (BLI)}

Tumor growth was monitored twice a week by bioluminescence imaging using an IVIS Spectrum Imaging System (Xenogen). An aqueous solution of luciferin (Caliper Life Sciences) at $150 \mathrm{mg} / \mathrm{kg}$ was injected intraperitoneal $10-20 \mathrm{~min}$ before imaging. Animals were anesthetized (2-4\% isoflurane) and placed in a dorsal position during imaging. Using the Living Image software 3.2 (Xenogen), photon flux was quantified within a circular region of interest (ROI) encompassing the head and neck region of each mouse. For 3D reconstruction, bioluminescence imaging (BLI) images were coregistered with computed tomography (CT) images.

\section{Fluorescence Molecular Tomography (FMT) Imaging}

One day prior to their endpoint and at least $20 \mathrm{~h}$ after the last HBOT session, mice were intravenously injected with $1.3 \mathrm{nmol}$ of the fluorescent blood pool imaging agent AngioSense750 (PerkinElmer) and/or $1.3 \mathrm{nmol}$ of the carbonic anhydrase IX (CAIX) targeted fluorescent imaging agent HypoxiSense680 (PerkinElmer) or MMPSense680, a probe that is activated after cleavage by matrix metalloproteinases (MMPs). For quantitative fluorescence molecular tomography imaging (FMT 2500, PerkinElmer), mice were anesthetized (2-4\% isoflurane) and fixed in a definite position in an animal imaging cassette. The FMT 2500 tomography software was used to quantitate fluorochrome concentration distribution of AngioSense in a ROI of $750 \mathrm{~mm}^{3}$ in the tumor area. In vivo imaging sessions were performed 2 and $24 \mathrm{~h}$ post-injection and hereafter, mice were euthanized (isoflurane overdose) and the tumors were dissected for ex vivo imaging. For multi-modality imaging, image data from FMT were fused with $\mathrm{CT}$ using markers in the multimodal mouse bed.

\section{Histology}

Mouse tumors were fixed in $10 \%$ formalin, embedded in paraffin, and $5 \mu \mathrm{m}$ slides were cut. Routine hematoxylin and eosin (H\&E) staining was performed and assessed by a pathologist. For immunohistochemistry, slides were probed with primary antibodies against Ki67 (Novus Biologicals Ltd.) and CD31 (Abcam) to assess proliferation and blood vessel density and diameter, respectively. Biotinylated goat anti-rabbit IgG (Dako) was used as secondary antibody, and detection was performed with streptavidin-peroxidase (R\&D Systems) and 3,3'-diaminobenzidine (Dako). Hematoxylin served as counterstain. Slides were scanned using a slide scanner (Hamamatsu Photonics). To measure proliferation, the percentage of Ki67-positive cells per tumor area was determined by using $\mathrm{Cell}^{\mathrm{d}}$ (Olympus Life Science Europe GmbH). Apoptosis levels were determined by counting the number of apoptotic cells in proliferating tumor areas $(\times 20)$ in H\&E-stained slides. To determine vascular density, CD31-positive blood vessels were counted in 20 representative fields $(\times 40)$ for each tumor. The vascular diameter of 30 vessels for each tumor was measured in $\times 63$ high power fields.

\section{Metastasis}

During the course of the experiment, the development of metastases was monitored by BLI of the total mouse body. Imaging was performed using unmixed emission spectra, allowing signal detection at particular tissue depths, which prevented outshining of the signal of the regional metastasis by the primary tumor. To establish the incidence of lymph node metastases, two superficial cervical lymph nodes were resected from each mouse, incubated for $10 \mathrm{~min}$ in luciferin solution (30 $\mu \mathrm{g} / \mathrm{ml})$, and ex vivo BLI was performed. To confirm the metastatic lesions, lymph nodes were embedded in paraffin, sectioned, and H\&E-stained.

\section{Quantitative Real-Time Reverse Transcription Polymerase Chain Reaction ( $q P C R$ )}

Mouse tumors were dissected, rapidly frozen in liquid nitrogen, and stored at $-80{ }^{\circ} \mathrm{C}$. Total RNA was isolated using the RNeasy Mini Kit (Qiagen) and reverse transcribed using the iScript cDNA Synthesis Kit (Bio-Rad). cDNA was amplified in 40 cycles $(20 \mathrm{~s}$ $95^{\circ} \mathrm{C}, 3 \mathrm{~s} 95^{\circ} \mathrm{C}, 30 \mathrm{~s} 60^{\circ} \mathrm{C}$ ) with a Bio-Rad cycler using Fast SYBR Green Master Mix (Applied Biosystems). Specific primers (see supplementary Table S1) were used to amplify cDNA of human vascular endothelial growth factor A (VEGF), carbonic anhydrase IX (CAIX), E-cadherin (CDH1), Vimentin (Vim), snail family zinc finger 1 (Snail), transforming growth factor beta 1 (TGF $\beta 1$ ), and the internal control glyceraldehyde-3-phosphate dehydrogenase $(\mathrm{GAPDH})$. Each PCR reaction was performed in duplicate, and the average threshold cycle $(\mathrm{Ct})$ value was used for relative quantification of gene expression with the comparative $\mathrm{Ct}$ method $(\Delta \Delta \mathrm{CT})$.

\section{Statistical Analysis}

Data are expressed as mean values with standard error of the mean (SEM), and were analyzed using SPSS PASW 21.0 for Windows (SPSS Inc., Chicago, USA). The Shapiro-Wilk test was used to test for normality, followed by the Mann-Whitney $U$ test for the comparison of non-normally distributed data, while Student's $t$ test was used for normally distributed data. $p<0.05$ indicated significant differences. Survival data were analyzed by the Kaplan-Meier and log-rank tests for survival distribution. The Fisher's exact test was used to analyze differences in the incidence of lymph node metastasis between groups. 
a

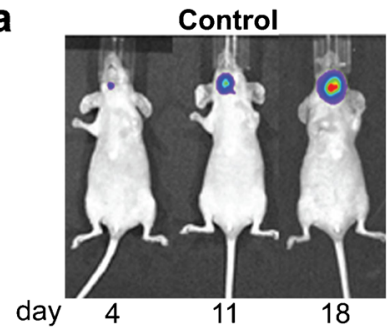

HBOT

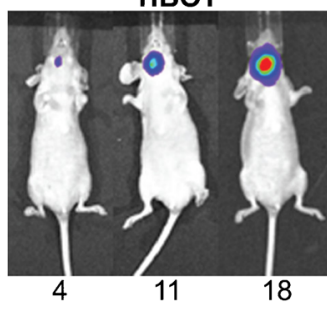

RT

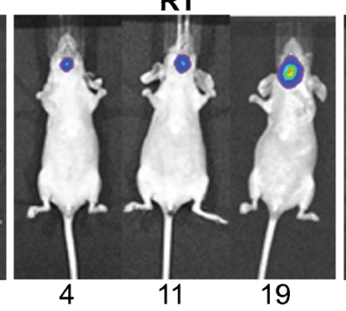

RT+HBOT

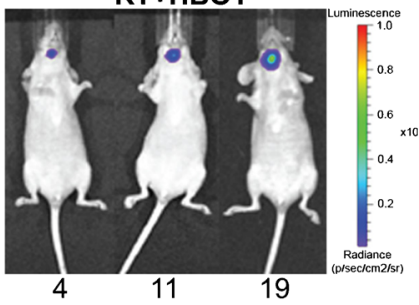

b

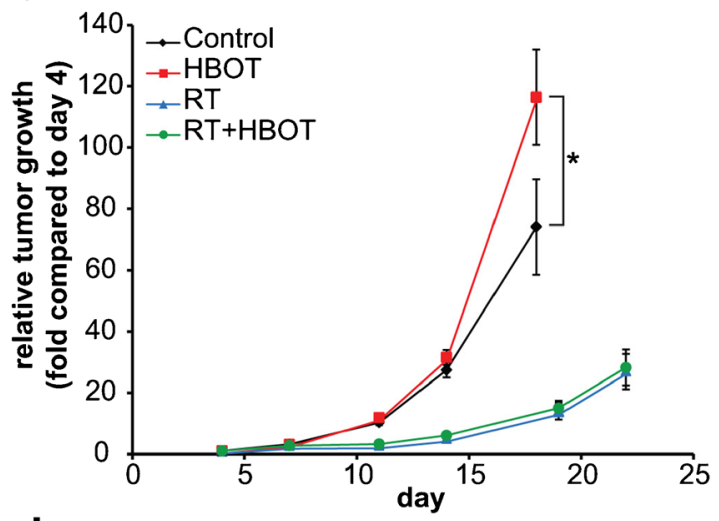

d

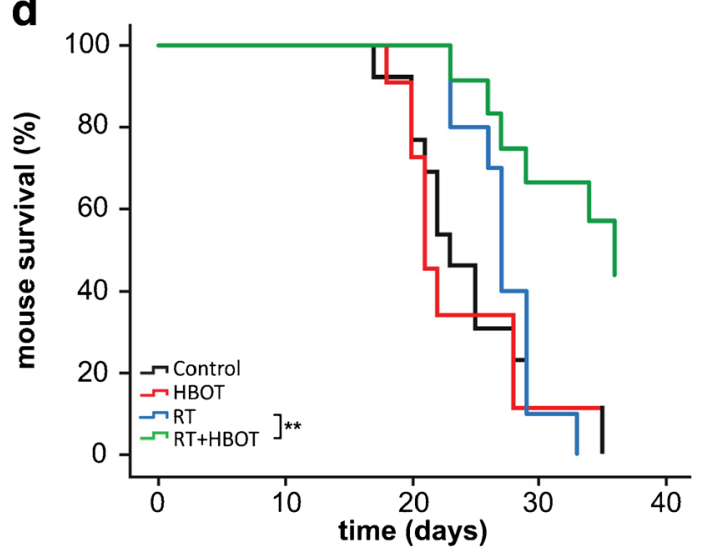

c
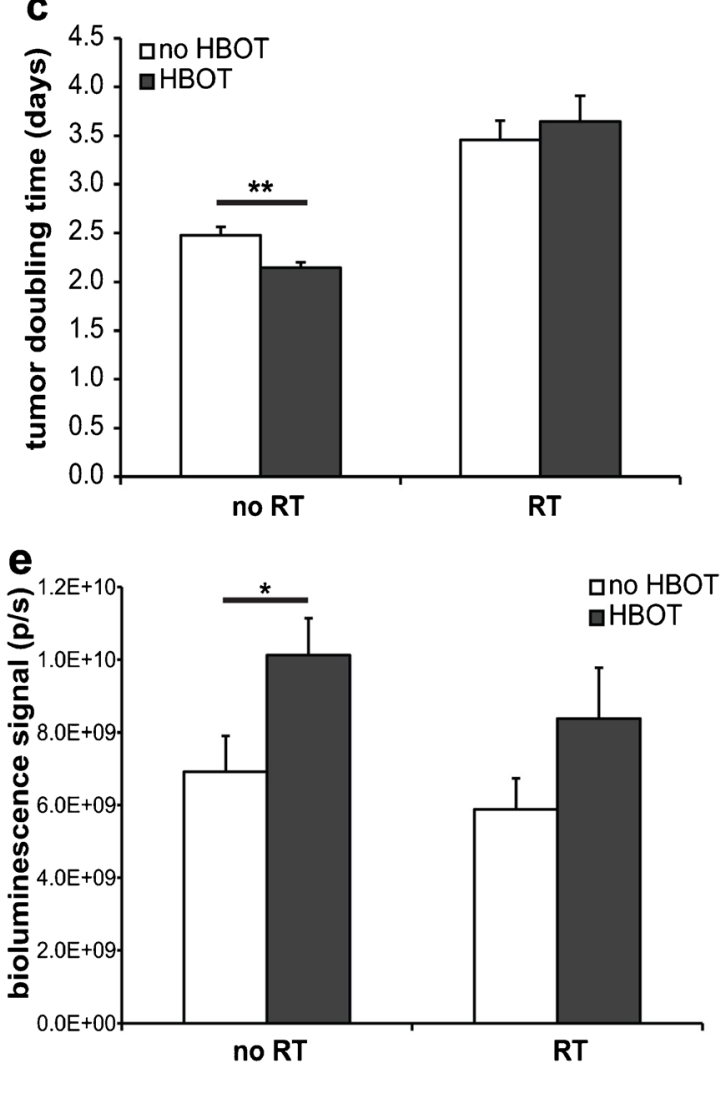

Fig. 1 In vivo effects of HBOT on tumor growth and survival time in FaDu-luc tumor-bearing mice. a Bioluminescence imaging (BLI) of representative mice of the different treatment groups on day 4, 11, and 18 or 19 after xenografting tumor cells in the floor of the mouth. $\mathbf{b}$ Increase of BLI signals compared to day 4 after xenografting tumor cells (9-12 mice per group). For the fold change numbers, see supplementary Table S2. c Mean doubling times of the tumors based on the BLI signals measured between day 7 and 18 (no RT) or day 11-22 (RT). d Mouse survival time analysis using the Kaplan-Meier method and comparisons using log-rank tests. e Mean BLI signals of the tumors at the endpoint. Error bars indicate SEM. ${ }^{\star *} p<0.01$; ${ }^{*} p<0.05$. $R T$ radiation therapy, HBOT hyperbaric oxygen therapy.

\section{Results}

\section{Effect of HBOT on Tumor Growth and Mouse Survival}

$\mathrm{FaDu}$ tumors were implanted in the floor of the mouth of nude mice, and the growth was monitored by bioluminescence imaging (BLI) until they met criteria for euthanasia, mostly due to weight loss. As shown in Fig. 1, the increase of the BLI signal was significantly higher $(p=0.023)$ in the group of mice that had undergone daily treatments of HBOT compared with the untreated group on day 18 after tumor cell inoculation (Fig. 1a, b). The mean doubling times for the
BLI signals of the individual tumors were significantly lower in the HBOT group versus the control (2.15 vs 2.47 days, $p=0.006$ ) (Fig. 1c). In irradiated tumors, the tumor growth rate was delayed compared to non-irradiated tumors, but no significant effect of HBOT was observed here (doubling times 3.46 and 3.65 days) (Fig. 1b, c).

The median survival periods for mice in the control, HBOT, RT, and RT+HBOT groups were 23, 21, 27, and 36 days, respectively. There was no significant effect of HBOT on the survival of non-irradiated mice, but mice with irradiated tumors had an increased survival time if HBOT had been applied ( $p=0.003)$ (Fig. 1d). The maximal BLI values measured at the time of euthanasia were higher in the 
HBOT group as compared to the control for both nonirradiated $(p=0.020)$ and irradiated tumors (not significant, $p=0.176$ ) (Fig. 1e).

\section{Effect of HBOT on Tumor Vascularization and Vascular Permeability}

Vascularization of the tumors was analyzed by investigating the CD31-positive blood vessels in tumor sections. The mean blood vessel density was slightly increased in irradiated tumors $(1.2$-fold, $p=0.014)$ but no significant effect of HBOT was observed (Fig. 2a). The mean tumor blood vessel diameter did not differ between the groups (Fig. 2b). mRNA levels of VEGF, a key factor involved in angiogenesis, were significantly increased in the irradiated tumors (1.3-fold, $p=0.000)$, but not affected by HBOT (Fig. 2c).

Tumor blood vessel quality was analyzed in vivo with FMT using AngioSense750 as a blood pool marker. AngioSense remains in the vasculature for $0-4 \mathrm{~h}$, and therefore the signal detected in the tumor area $2 \mathrm{~h}$ after probe injection is a measure for the tumor vascular volume. The degree of AngioSense retention in the tumor area after $24 \mathrm{~h}$ is indicative for vascular leakiness [25, 26]. In Fig. 2d, the site of accumulation of AngioSense750 in and around the tumor is shown and coregistered with the signal of the probe MMPSense680 which indicates the tumor margins. For each individual mouse, the ratio between the $24 \mathrm{~h}$ and $2 \mathrm{~h}$ AngioSense signal was determined and probe accumulation appeared to be higher in the mice treated with HBOT in both the non-irradiated (1.3-fold, $p=0.042)$ and the irradiated a

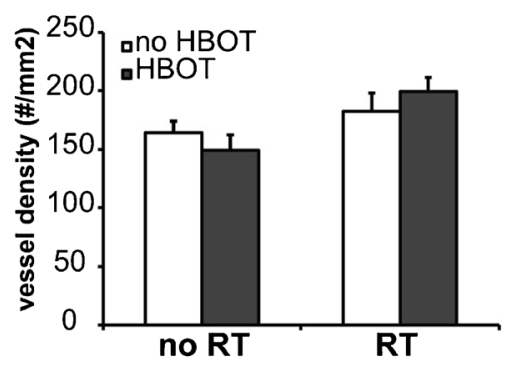

b

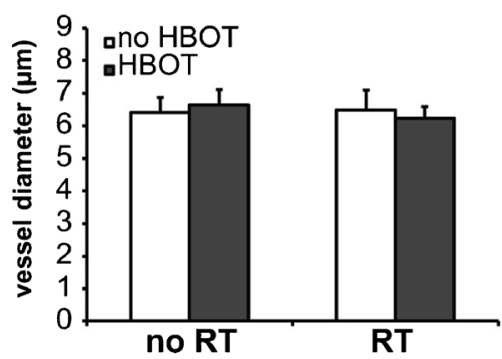

C

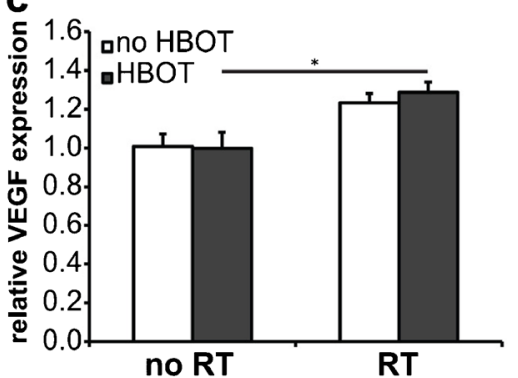

d

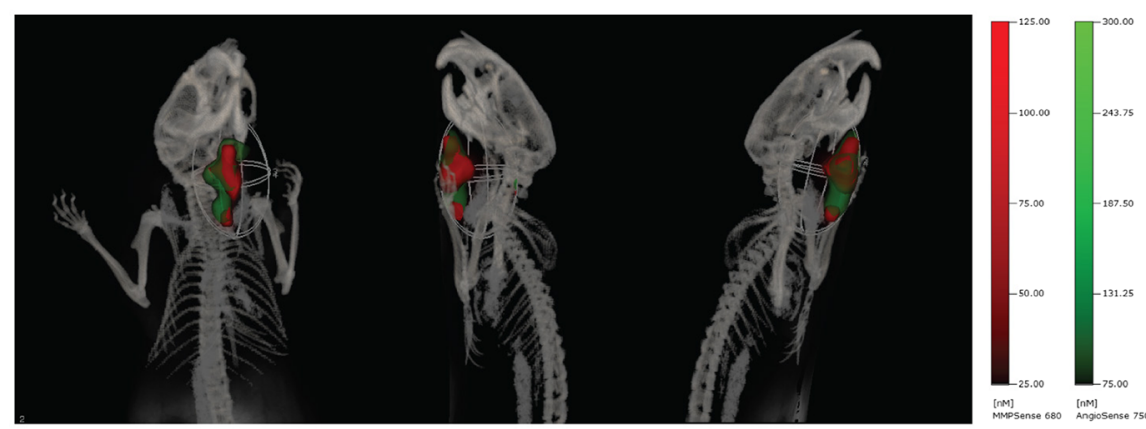

e

Control

HBOT

$2 \mathrm{~h}$
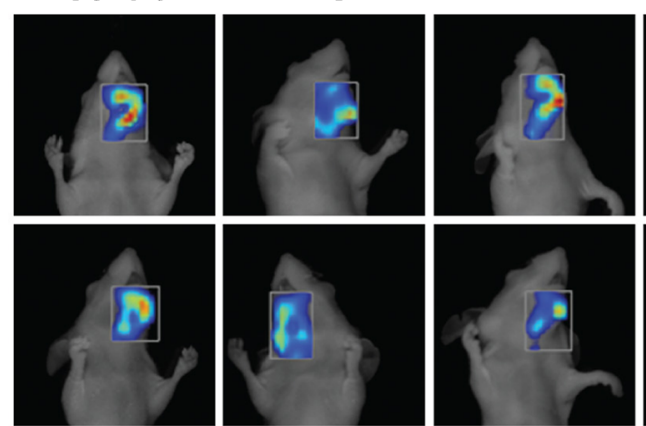

RT+HBOT

$24 \mathrm{~h}$
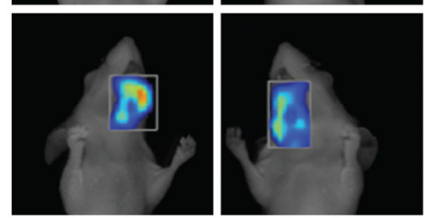

$\mathbf{f}$

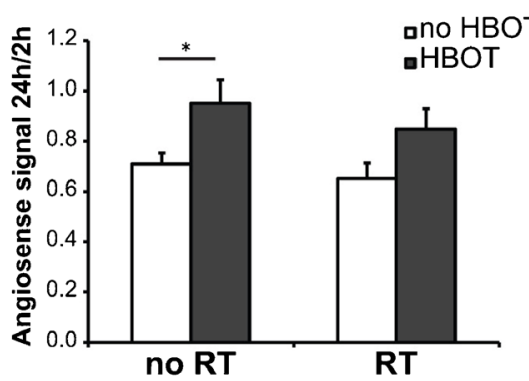

Fig. 2 Effect of HBOT on tumor vasculature. a Quantification of the tumor blood vessel density. $\mathbf{b}$ Quantification of the tumor blood vessel diameter. $\mathbf{c}$ Relative expression levels of VEGF mRNA in the tumors. d Multimodal FMT/CT imaging of a FaDu-luc mouse $24 \mathrm{~h}$ after injection of MMPSense680 (red) and AngioSense750 (green) to detect tumor margins and region of tumor vascular leak, respectively. e Representative FMT images of tumor regions in FaDu-luc mice of the different treatment groups, 2 and $24 \mathrm{~h}$ after AngioSense750 injection. $\mathbf{f}$ Quantification of blood vessel leakage in the tumor regions. For each animal, the $24 \mathrm{~h} / 2 \mathrm{~h}$ AngioSense signal ratio was determined $(n=6)$. Error bars indicate SEM. ${ }^{*} p<0.05$. $R T$ radiation therapy, HBOT hyperbaric oxygen therapy. 
group (1.3-fold, $p=0.078$ ), indicating an increase in tumor vascular permeability after HBOT [Fig. 2e, f].

\section{Effect of HBOT on Tumor Hypoxia}

In tumors of irradiated animals, a clear increase in mRNA levels of the hypoxia inducible factor CAIX (2.4-fold, $p=$ 0.000 ) was observed, but no significant effect of HBOT on CAIX expression was detected (Fig. 3a).

Tumor hypoxia was further analyzed by FMT using the CAIX targeted fluorescent imaging agent HypoxiSense680 as a probe. Because of the low fluorescence levels, it was not possible to obtain in vivo data regarding the hypoxic state of the tumors. Mice were sacrificed $24 \mathrm{~h}$ after probe injection and analyzed ex vivo (Fig. 3b). HypoxiSense signals were detected in none of the control tumors but half of the HBOT tumors. Stronger HypoxiSense signals were observed in the tumors of the irradiated animals with again the highest fluorescent levels in the HBOT group (Fig. 3b, c), indicating that tumor hypoxia is increased after HBOT.

\section{Effect of HBOT on Tumor Pathological Features and Metastasis}

The tumors were rated as poorly to moderately differentiated squamous cell carcinoma with moderate infiltrative borders for all experimental groups (Fig. 4a, b, c). Perineural growth and vascular invasion of tumor cells was evident in several tumor sections but no significant differences were observed between the HBOT and the control groups. The degree of necrosis (Table 1$)$ was highly variable among the tumors $(0-$ $42.2 \%$ of the tumor area) and was related to tumor size, but no significant effect of HBOT was established. Tumor cell proliferation and apoptosis levels were also not significantly affected by HBOT although a trend towards decreased cell death after HBOT was observed (Table 1).
To investigate the impact of HBOT on epithelial-tomesenchymal transition (EMT), mRNA expression levels of the malignancy markers E-cadherin, Vimentin, Snail, and TGF $\beta 1$ were determined. Vimentin expression was slightly upregulated in the irradiated tumors (1.4-fold, $p=0.039)$ but its levels were not significantly influenced by HBOT (Fig. 4e). On the tumor expression of E-cadherin, Snail, and TGF $\beta 1$, no significant effects of either RT or HBOT were observed (Fig. 4d, f, g).

The orthotopic tumor model allowed us to identify and monitor the development of regional or distant metastases in real time by in vivo BLI using spectral unmixing and 3D reconstruction to circumvent outshining of the signals by the strong total BLI signals of the primary tumor in the floor of the mouth (Fig. 4h and Online video). In the time frame of the experiment, distant metastases were not detected but cervical lymph node metastases developed in the majority of the mice. To confirm and characterize the metastases, two superficial cervical lymph nodes of each mouse were harvested immediately after euthanasia and analyzed by ex vivo BLI and histology (Fig. 4i, j). For the non-irradiated as well as the irradiated mice, the metastatic incidence was not affected by HBOT (Table 2). Also, no effect of HBOT on the histological stage of the lymph node metastases was observed.

\section{Discussion}

In this study, tumor responses to HBOT were investigated in an orthotopic mouse model of head and neck squamous cell carcinoma using optical imaging methods. By means of bioluminescence imaging, the growth of a human hypopharyngeal carcinoma cell line in the floor of the mouth of mice was accurately monitored and revealed a small but significant increase in tumor growth rate (19\%) under the influence of HBOT. No effect of HBOT, however, was detected on the growth of tumors that had been irradiated before. The difference in response might lie in a

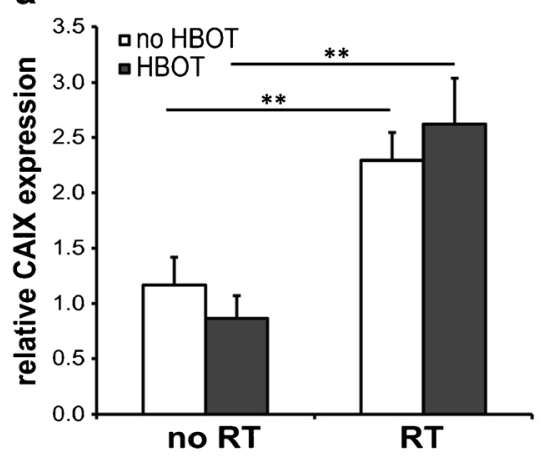

b

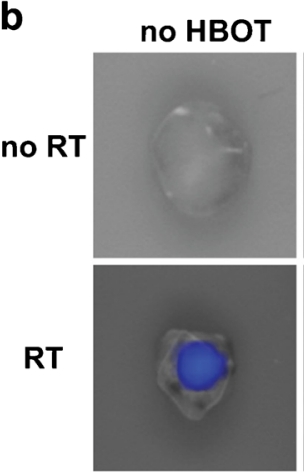

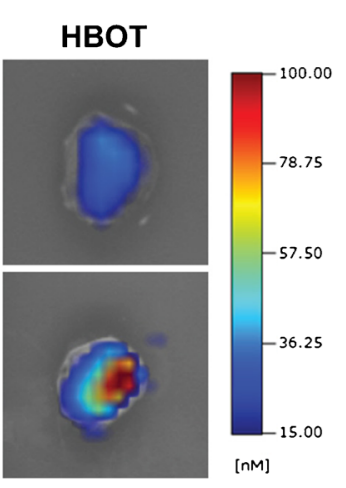

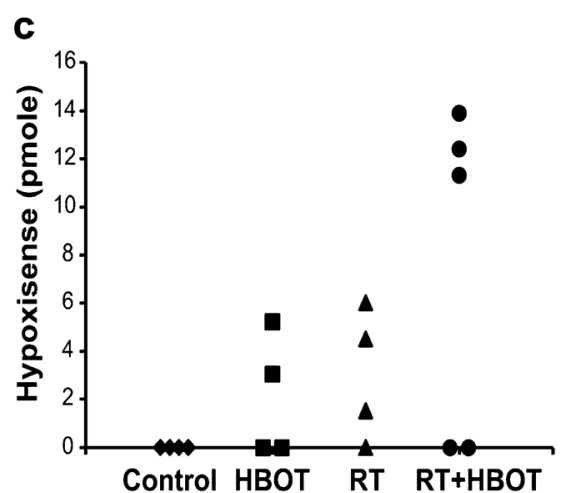

Fig. 3 Effect of HBOT on tumor hypoxia. a Relative expression levels of CAIX mRNA in the tumors as determined by qPCR. Error bars indicate SEM. ${ }^{* *} p<0.01$. b Representative ex vivo FMT images of dissected FaDu-luc tumors $24 \mathrm{~h}$ after HypoxiSense680 injection. c Quantification of HypoxiSense signals in individual dissected tumors. $R T$ radiation therapy, HBOT hyperbaric oxygen therapy. 

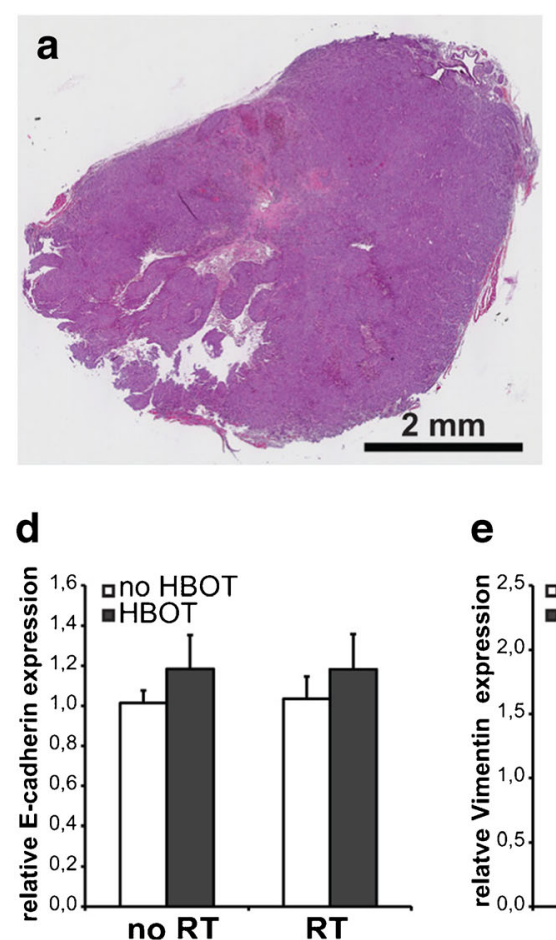

h

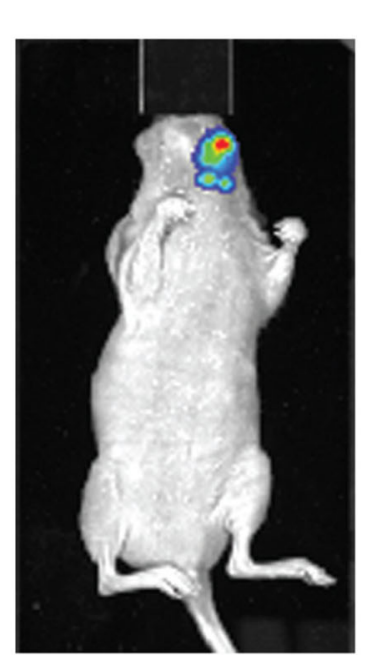

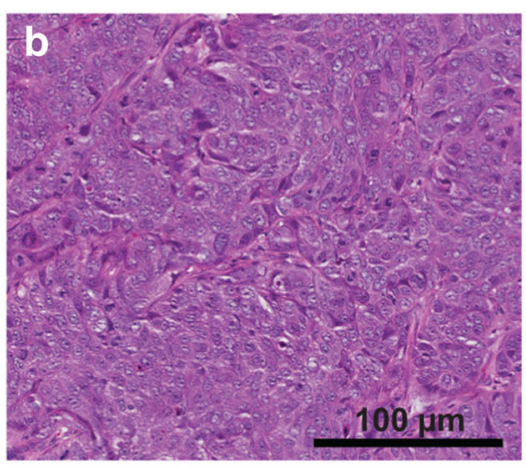

f
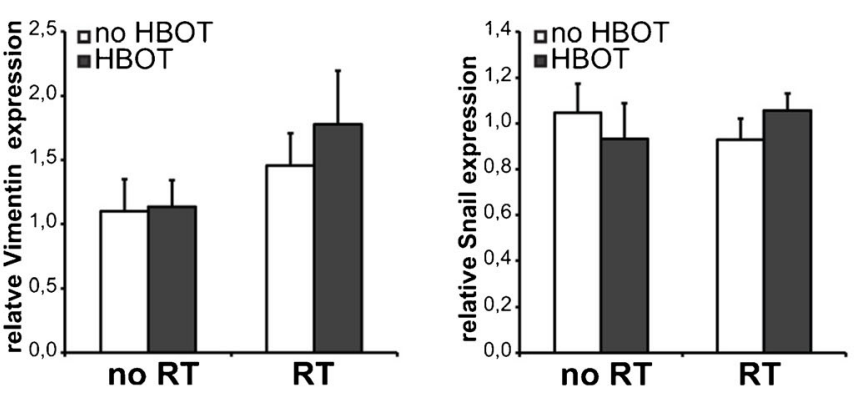

g
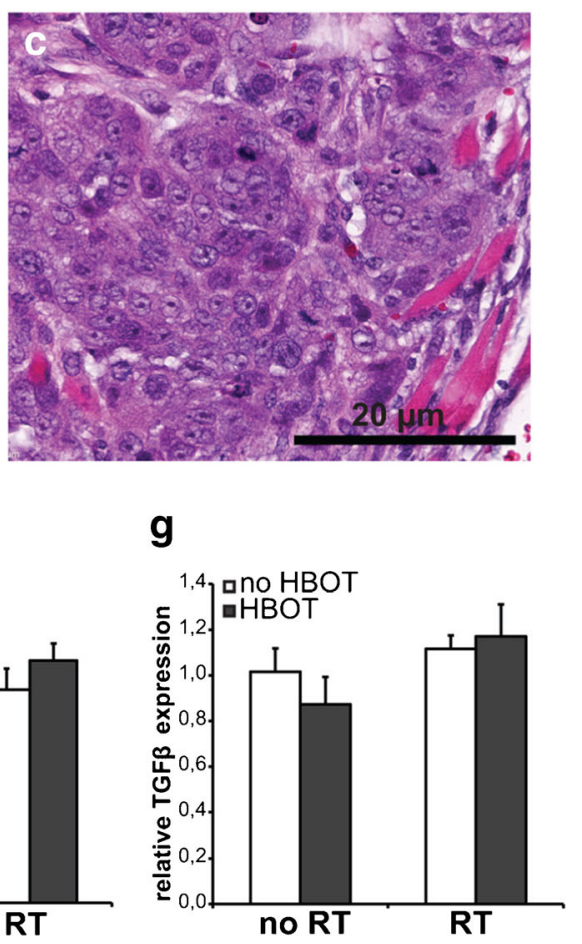

i
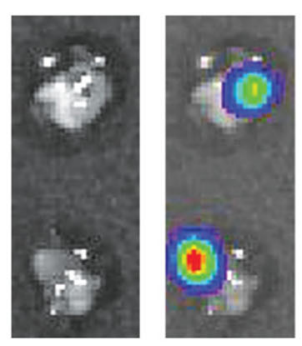

Fig. 4 Effect of HBOT on tumor malignancy parameters. a H\&E staining of a tissue section of a representative FaDu-luc tumor dissected from the floor of the mouth. All treatment groups show poorly to moderately differentiated squamous cell carcinoma. b Detail of A. c Detail of A. d-g Relative expression levels of E-cadherin (d), Vimentin (e), Snail (f), and TGF 31 (g) mRNA in the tumors. $R T$ radiation therapy, HBOT hyperbaric oxygen therapy. $\mathbf{h} / n$ vivo $\mathrm{BLI}$ of a tumor-bearing mouse at day 20 at an emission wavelength of $560 \mathrm{~nm}$ showing lymph node metastases. i Photographic (left) and bioluminescent (right) images of metastasized cervical lymph nodes. $\mathbf{j}$ H\&E staining of a tissue section of a representative metastasized lymph node.

Table 1. Proliferation, apoptosis, and necrosis in FaDu tumors

\begin{tabular}{llccc}
\hline Factor & Control & HBOT & RT & RT+HBOT \\
\hline Proliferation (\% Ki67-positive tumor cells) $^{\mathrm{a}}$ & $38.1 \pm 1.8$ & $40.0 \pm 1.5$ & $43.2 \pm 1.7$ & $41.9 \pm 1.2$ \\
Apoptosis (\# cells/mm $\left.^{2}\right)^{\mathrm{a}}$ & $30.6 \pm 2.9$ & $24.9 \pm 2.4$ & $22.2 \pm 3.9$ & $19.0 \pm 4.1$ \\
Necrosis (\% area) $^{\mathrm{b}}$ & $2.9[0-28.0]$ & $6.9[0.5-27.5]$ & $5.0[0-42.1]$ & $6.9[0-31.0]$ \\
\hline
\end{tabular}

$R T$ radiation therapy, HBOT hyperbaric oxygen therapy

${ }^{\mathrm{a}}$ Mean values \pm SEM

${ }^{\mathrm{b}}$ Median values [range] 
Table 2. Incidence and rate of lymph node metastasis in mice with $\mathrm{FaDu}$ tumors

\begin{tabular}{llclc}
\hline Factor & Control & HBOT & RT & $\begin{array}{l}\text { RT }+ \\
\text { HBOT }\end{array}$ \\
\hline Metastatic incidence & $8 / 12$ & $8 / 11$ & $9 / 10$ & $10 / 11$ \\
Metastatic percentage & $67 \%$ & $72 \%$ & $90 \%$ & $91 \%$ \\
$\begin{array}{l}\text { Fisher's exact test vs } \\
\text { control }\end{array}$ & & 1.000 & 0.323 & 0.317 \\
\hline
\end{tabular}

$R T$ radiation therapy, HBOT hyperbaric oxygen therapy

the fact that irradiation, in addition to killing tumor cells, also damages endothelial cells $[27,28]$, resulting in a tumor microenvironment that is less susceptible to HBOT-induced, growth promoting stimuli. Although tumors grew faster in HBOT-treated mice as compared to controls, the survival time of these animals was not affected. Interestingly, the bioluminescent signals of the tumors at the endpoints were higher in the HBOT group, indicating that these mice survived higher loads of viable tumor tissue. Also, in the irradiated groups, in which the survival period was extended by HBOT but the tumor growth rate was not affected, a trend towards increased endpoint tumor load was noticed. HBOT might affect the disturbed metabolic processes in the body that lead to cancer-associated weight loss and in this way increase survival rates. From a clinical point of view, this is an interesting finding and future studies are warranted to investigate this potential beneficial impact of HBOT. Histological analyses could not reveal the factors that underlie the accelerated tumor growth after HBOT. Small and possibly transient influences of HBOT on vascularization, proliferation, or cell death might have lead to the observed growth effect. HBOT stimulates vessel development in normal tissue and in wounds $[3,5]$ but its effect on tumor vascularization is unclear. Tumors possess disorganized and leaky tumor vessels which block adequate tissue perfusion leading to the presence of hypoxic regions that are associated with poor prognosis and treatment outcome [29, 30]. Normalization of the tumor vasculature is thought to lead to less tumor hypoxia and is a goal of anti-angiogenic therapies [9, 31]. In our HNSCC tumor model, in vivo molecular imaging with the blood pool agent AngioSense disclosed a higher vascular leakiness in tumors of HBOTtreated animals. This is the first study exploring the effects of HBOT on vascular permeability, indicating that HBOT does not lead to normalization of blood vessels and might even deteriorate tumor vascular quality.

To compare the hypoxic states of the tumors, the recently developed HypoxiSense probe, which detects the protein CAIX on the tumor cell surface, was employed. Due to relatively low fluorescent signals, however, in vivo data could not be obtained. In previous studies, this fluorescent agent was successfully used in subcutaneous xenograft tumors with volumes of $600-700 \mathrm{~mm}[3,32]$. In our orthotopic model, the tumors in the floor of the mouth did not grow beyond $250 \mathrm{~mm}^{3}$ and therefore signal detection was probably hampered by optical properties such as background absorption and scattering [33]. Nevertheless, ex vivo, hypoxic regions were detected in a subset of tumors and the data suggest that irradiated tumors were more hypoxic than non-irradiated tumors and moreover, that HBOT aggravated tumor hypoxia as well. It has been demonstrated that HBOT increases the oxygen concentration in tumor tissue during and shortly after treatment but this effect is transient [34, 35]. The drop in oxygen level following a HBOT session may lead to the induction of a hypoxic response in the tumor tissue, by which CAIX expression could be enhanced. This would correspond to previous studies in which exposure to HBOT resulted in increased levels of the hypoxia inducible factor HIF-1a in the liver and brain of rats [36-38]. It is also possible that CAIX production was not stimulated by hypoxia, but by inflammatory cytokines that are known for their ability to induce HIF-1a stabilization as well [39]. The fact that CAIX mRNA levels were not found to be elevated in the dissected tumors suggests that CAIX gene activation had been transient, and that transcription had returned to normal levels since the last HBOT session. In conclusion, our data indicate that the use of intensive HBOT protocols does not lead to long-term overall reduction of tumor hypoxic responses.

The presence of cervical lymph node metastasis is an important prognostic indicator for patients with HNSCC. The current bioluminescent orthotopic tumor model allowed us to monitor the consequences of treatment for the development of lymph node metastases. Metastatic incidence was increased from approximately 70 to $90 \%$ in the irradiated animals, but was not affected by HBOT. This confirms previous experimental results obtained in different cancer and animal models, in which stimulation of metastasis by HBOT was not established either [16, 40-44]. Histopathological and molecular analysis of the tumors did not reveal significant HBOT-induced changes in malignant parameters. Epithelial-to-mesenchymal transition (EMT) is a central mechanism of cancer metastasis whereby tumor cells are reprogrammed, resulting in decreased adhesion and enhanced migration and invasion [45-47]. The expression of the hallmark molecules of EMT, the epithelial marker Ecadherin, the mesenchymal marker Vimentin, and the EMTinducing factors Snail and TGF $\beta 1$ was not affected by HBOT, indicating that there was no switch to more aggressive tumors. Moen et al. [15] reported induction of mesenchymal-to-epithelial transition (MET) by HBOT in a mammary tumor model, but thus far there are no indications for similar effects in squamous cell cancer.

Altogether, in this study, we found that HBOT stimulated the growth of non-irradiated tumors and increased tumor blood vessel leakiness and hypoxia. These are factors known to promote aggressive tumor behavior and poorer treatment outcome $[10,30]$. On the other hand, HBOT was beneficial for animal survival and no effects of HBOT were detected on metastatic incidence, histological grade, and malignancy 
markers, suggesting that the effects of HBOT on disease outcome are limited and there might be no increased risk for negative effects of HBOT in patients that were previously subjected to radiation therapy. Previous experimental studies on the effects of HBOT on tumor behavior thus far yielded varying results $[8,11]$. In mammary and glioma tumor models, growth-inhibiting and anti-angiogenic effects were reported using a HBOT schedule of 3 to 4 sessions per week $[13,14,48]$. Studies using squamous cell cancer models did not reveal effects of HBOT on tumor growth [16-20], except for a recent study by Paniello et al. [21] who also observed enhanced growth of xenografted HNSCC tumors in mice. In these studies, daily HBOT sessions were applied, according to the clinically used protocols for the management of radiation-induced injury. Therefore, specific tumor characteristics and HBOT time schedules might underlie the different outcomes and need to be considered in future studies.

\section{Conclusions}

The improved animal model and in vivo molecular imaging methods used in this study disclosed influences of HBOT on the growth rate, blood vessel quality, and hypoxic state of squamous cell carcinoma and opens up possibilities to further investigate the circumstances and conditions in which HBOT can be safely used in cancer patients.

Acknowledgments. Isabel Mol and Paula van Heijningen are gratefully acknowledged for their technical assistance. This work was supported by the Applied Molecular Imaging Erasmus MC (AMIE) facility by providing the imaging equipment. This research was financially supported by Fonds NutsOhra (grant number 1101-018), ZonMw medium-sized equipment grant (grant number 40-00506-98-11012), and "Stichting Lijf en Leven ."

Ethical Approval. All applicable institutional and/or national guidelines for the care and use of animals were followed.

Conflict of Interest. The authors declare that they have no conflict of interest.

Open Access This article is distributed under the terms of the Creative Commons Attribution License which permits any use, distribution, and reproduction in any medium, provided the original author(s) and the source are credited.

\section{References}

1. Spiegelberg L, Djasim UM, van Neck HW et al (2010) Hyperbaric oxygen therapy in the management of radiation-induced injury in the head and neck region: a review of the literature. J Oral Maxillofac Surg 68:1732-1739

2. Bennett MH, Feldmeier J, Hampson N et al (2012) Hyperbaric oxygen therapy for late radiation tissue injury. Cochrane Database Syst Rev 5, CD005005

3. Hopf HW, Gibson JJ, Angeles AP et al (2005) Hyperoxia and angiogenesis. Wound Repair Regen 13:558-564

4. Thom SR (2011) Hyperbaric oxygen: its mechanisms and efficacy. Plast Reconstr Surg 127(Suppl 1):131S-141S

5. Drenjancevic I, Kibel A (2014) Restoring vascular function with hyperbaric oxygen treatment: recovery mechanisms. J Vasc Res 51:113
6. Feldmeier J, Carl U, Hartmann K, Sminia P (2003) Hyperbaric oxygen: does it promote growth or recurrence of malignancy? Undersea Hyperb Med 30:1-18

7. Moon RE, Feldmeier JJ (2002) Hyperbaric oxygen: an evidence based approach to its application. Undersea Hyperb Med 29:1-3

8. Wenwu L, Xuejun S, Hengyi T, Kan L (2013) Hyperbaric oxygen and cancer: more complex than we expected. Target Oncol 8:79-81

9. Michieli P (2009) Hypoxia, angiogenesis and cancer therapy: to breathe or not to breathe? Cell Cycle 8:3291-3296

10. Vaupel P, Mayer A (2007) Hypoxia in cancer: significance and impact on clinical outcome. Cancer Metastasis Rev 26:225-239

11. Moen I, Stuhr LE (2012) Hyperbaric oxygen therapy and cancer-a review. Target Oncol 7:233-242

12. Daruwalla J, Christophi C (2006) Hyperbaric oxygen therapy for malignancy: a review. World J Surg 30:2112-2131

13. Raa A, Stansberg C, Steen VM et al (2007) Hyperoxia retards growth and induces apoptosis and loss of glands and blood vessels in DMBAinduced rat mammary tumors. BMC Cancer 7:23

14. Stuhr LE, Raa A, Oyan AM et al (2007) Hyperoxia retards growth and induces apoptosis, changes in vascular density and gene expression in transplanted gliomas in nude rats. J Neurooncol $85: 191-202$

15. Moen I, Oyan AM, Kalland KH et al (2009) Hyperoxic treatment induces mesenchymal-to-epithelial transition in a rat adenocarcinoma model. PLoS One 4:e6381

16. Schonmeyr BH, Wong AK, Reid VJ et al (2008) The effect of hyperbaric oxygen treatment on squamous cell cancer growth and tumor hypoxia. Ann Plast Surg 60:81-88

17. Sklizovic D, Sanger JR, Kindwall EP et al (1993) Hyperbaric oxygen therapy and squamous cell carcinoma cell line growth. Head Neck $15: 236-240$

18. Headley DB, Gapany M, Dawson DE et al (1991) The effect of hyperbaric oxygen on growth of human squamous cell carcinoma xenografts. Arch Otolaryngol Head Neck Surg 117:1269-1272

19. Shi Y, Lee CS, Wu J et al (2005) Effects of hyperbaric oxygen exposure on experimental head and neck tumor growth, oxygenation, and vasculature. Head Neck 27:362-369

20. Sun TB, Chen RL, Hsu YH (2004) The effect of hyperbaric oxygen on human oral cancer cells. Undersea Hyperb Med 31:251-260

21. Paniello RC, Fraley PL, O’Bert R (2014) Effect of hyperbaric oxygen therapy on a murine squamous cell carcinoma model. Head Neck 36:1743-1746

22. Wachsberger P, Burd R, Dicker AP (2003) Tumor response to ionizing radiation combined with antiangiogenesis or vascular targeting agents: exploring mechanisms of interaction. Clin Cancer Res 9:1957-1971

23. Keereweer S, Mol IM, Vahrmeijer AL et al (2012) Dual wavelength tumor targeting for detection of hypopharyngeal cancer using nearinfrared optical imaging in an animal model. Int J Cancer 131:16331640

24. Djasim UM, Spiegelberg L, Wolvius EB, van der Wal KG (2012) A hyperbaric oxygen chamber for animal experimental purposes. Int $\mathrm{J}$ Oral Maxillofac Surg 41:271-274

25. Tseng JC, Granot T, DiGiacomo V et al (2010) Enhanced specific delivery and targeting of oncolytic Sindbis viral vectors by modulating vascular leakiness in tumor. Cancer Gene Ther 17:244-255

26. Ackermann M, Carvajal IM, Morse BA et al (2011) Adnectin CT-322 inhibits tumor growth and affects microvascular architecture and function in Colo205 tumor xenografts. Int J Oncol 38:71-80

27. Shinohara ET, Geng L, Tan J et al (2005) DNA-dependent protein kinase is a molecular target for the development of noncytotoxic radiation-sensitizing drugs. Cancer Res 65:4987-4992

28. Budach W, Taghian A, Freeman J et al (1993) Impact of stromal sensitivity on radiation response of tumors. J Natl Cancer Inst 85:988-993

29. Ribatti D, Nico B, Crivellato E, Vacca A (2007) The structure of the vascular network of tumors. Cancer Lett 248:18-23

30. McDonald DM, Baluk P (2002) Significance of blood vessel leakiness in cancer. Cancer Res 62:5381-5385

31. Jain RK (2013) Normalizing tumor microenvironment to treat cancer: bench to bedside to biomarkers. J Clin Oncol 31:22052218

32. Bao B, Groves $\mathrm{K}$, Zhang $\mathrm{J}$ et al (2012) In vivo imaging and quantification of carbonic anhydrase IX expression as an endogenous biomarker of tumor hypoxia. PLoS One 7:e50860 
33. Keereweer S, Van Driel PB, Snoeks TJ et al (2013) Optical imageguided cancer surgery: challenges and limitations. Clin Cancer Res 19:3745-3754

34. Kinoshita Y, Kohshi K, Kunugita N et al (2000) Preservation of tumour oxygen after hyperbaric oxygenation monitored by magnetic resonance imaging. Br J Cancer 82:88-92

35. Brizel DM, Lin S, Johnson JL et al (1995) The mechanisms by which hyperbaric oxygen and carbogen improve tumour oxygenation. Br J Cancer 72:1120-1124

36. Salhanick SD, Belikoff B, Orlow D et al (2006) Hyperbaric oxygen reduces acetaminophen toxicity and increases HIF-1alpha expression. Acad Emerg Med 13:707-714

37. Ren P, Kang Z, Gu G et al (2008) Hyperbaric oxygen preconditioning promotes angiogenesis in rat liver after partial hepatectomy. Life Sci 83:236-241

38. Gu GJ, Li YP, Peng ZY et al (2008) Mechanism of ischemic tolerance induced by hyperbaric oxygen preconditioning involves upregulation of hypoxia-inducible factor-1alpha and erythropoietin in rats. J Appl Physiol 104:1185-1191

39. Kockar F, Yildrim H, Sagkan RI et al (2012) Hypoxia and cytokines regulate carbonic anhydrase 9 expression in hepatocellular carcinoma cells in vitro. World J Clin Oncol 3(6):82-91

40. Poff AM, Ari C, Seyfried TN, D'Agostino DP (2013) The ketogenic diet and hyperbaric oxygen therapy prolong survival in mice with systemic metastatic cancer. PLoS One 8:e65522
41. Petre PM, Baciewicz FA Jr, Tigan S, Spears JR (2003) Hyperbaric oxygen as a chemotherapy adjuvant in the treatment of metastatic lung tumors in a rat model. J Thorac Cardiovasc Surg 125:85-95, discussion 95

42. Kawasoe Y, Yokouchi M, Ueno Y et al (2009) Hyperbaric oxygen as a chemotherapy adjuvant in the treatment of osteosarcoma. Oncol Rep 22:1045-1050

43. Haroon AT, Patel M, Al-Mehdi AB (2007) Lung metastatic load limitation with hyperbaric oxygen. Undersea Hyperb Med 34:83-90

44. Daruwalla J, Christophi C (2006) The effect of hyperbaric oxygen therapy on tumour growth in a mouse model of colorectal cancer liver metastases. Eur J Cancer 42:3304-3311

45. Lee JM, Dedhar S, Kalluri R, Thompson EW (2006) The epithelialmesenchymal transition: new insights in signaling, development, and disease. J Cell Biol 172:973-981

46. Yang J, Weinberg RA (2008) Epithelial-mesenchymal transition: at the crossroads of development and tumor metastasis. Dev Cell $14: 818-829$

47. Chaffer CL, Thompson EW, Williams ED (2007) Mesenchymal to epithelial transition in development and disease. Cells Tissues Organs 185:7-19

48. Moen I, Tronstad KJ, Kolmannskog O et al (2009) Hyperoxia increases the uptake of 5-fluorouracil in mammary tumors independently of changes in interstitial fluid pressure and tumor stroma. BMC Cancer 9:446 